\title{
Orienting of attention, pupil size, and the norepinephrine system
}

\author{
Shai Gabay • Yoni Pertzov • Avishai Henik
}

Published online: 24 November 2010

(C) Psychonomic Society, Inc. 2010

\begin{abstract}
This research examined a novel suggestion regarding the involvement of the locus coeruleus-norepinephrine (LC-NE) system in orienting reflexive (exogenous) attention. A common procedure for studying exogenous orienting of attention is Posner's cuing task. Importantly, one can manipulate the required level of target processing by changing task requirements, which, in turn, can elicit a different time course of inhibition of return (IOR). An easy task (responding to target location) produces earlier onset IOR, whereas a demanding task (responding to target identity) produces later onset IOR. Aston-Jones and Cohen (Annual Review of Neuroscience, 28, 403-450, 2005) presented a theory suggesting two different modes of LC activity: tonic and phasic. Accordingly, we suggest that in the more demanding task, the LC-NE system is activated in phasic mode, and in the easier task, it is activated in tonic mode. This, in turn, influences the appearance of IOR. We examined this suggestion by measuring participants' pupil size, which has been demonstrated to correlate with the LC-NE system, while they performed cuing tasks. We found a response-locked phasic dilation of the pupil in the discrimination task, as compared with the localization task, which may reflect different firing modes of the LC-NE system during the two tasks. We also demonstrated a correlation between pupil size at the time of cue presentation and magnitude of IOR.
\end{abstract}

\section{S. Gabay $(\triangle) \cdot$ A. Henik}

Department of Psychology and Zlotowski Center for Neuroscience, Ben-Gurion University of the Negev, P.O. Box 653, Beer-Sheva, Israel 84105

e-mail: shaigaba@bgu.ac.il

\section{Y. Pertzov}

Interdisciplinary Center for Neural Computation and the Department of Neurobiology, Alexander Silberman Institute of Life Sciences, Hebrew University of Jerusalem,

Jerusalem, Israel 91904

\section{Keywords IOR $\cdot$ Pupil size $\cdot$ Spatial attention $\cdot$ LC-NE}

Task demands can influence our ability to process irrelevant stimuli. For instance, when driving on a busy highway during rush hours and trying to safely navigate in the heavy traffic, we would be less sensitive to what the passenger next to us is saying or doing as compared with when driving during nonrush hours. Recent work by Aston-Jones and Cohen (2005) suggested that task demands might modulate alertness through activation of the locus coeruleus-norepinephrine (LC-NE) system. Alertness, in turn, might modulate orienting. Accordingly, this work examines the influence of task demands on orienting of attention. This is done by measuring pupil size, which is suggested to be correlated with the performance of the LC-NE system.

\section{Orienting of attention}

Posner's cuing task (Posner \& Cohen, 1984) enables examining endogenous (volitional) and exogenous (reflexive) orienting of attention. When endogenous orienting is studied, a predictive central cue is presented before the appearance of a target. The typical pattern of results elicited in such conditions is a gradually developing validity effect. That is, reaction time (RT) is shorter for valid trials (i.e., target and cue appear at the same location) than for invalid trials (i.e., target and cue appear at opposite locations). When exogenous orienting is studied, a nonpredictive peripheral cue is presented before the appearance of the target. The typical pattern of results is an early validity effect, followed by inhibition of return (IOR). That is, RT is shorter for valid trials than for invalid trials at short stimulus onset asynchronies (SOAs; the duration from cue 
onset until target onset) and longer for valid than for invalid trials at longer SOAs.

It was originally assumed that IOR does not appear in discrimination tasks (Terry, Valdes, \& Neill, 1994), but later work by Lupiáñez, Milan, Tornay, Madrid, and Tudela (1997) has shown that the onset of IOR develops later in discrimination tasks than in detection tasks. The difference in the onset of the IOR effect elicited by the detection versus discrimination tasks may be related to the recruitment of different processes or to the amount of attention they require. For example, Egly and Homa (1991) suggested that simpler tasks such as detection may not recruit the additional processes that more demanding tasks do. Klein (2000) suggested that in a more difficult task (discrimination), participants allocate more attention to all the stimuli (target and cue) and that the longer focus of attention at the cued location delays the appearance of IOR (see also Berger, Henik, \& Rafal, 2005). However, the differences between tasks may reflect differences in other attentional systems (e.g., alertness). The interaction between orienting of attention and other attentional systems, such as alertness, has attracted much interest recently, and we discuss this issue below.

\section{Alertness and other attentional systems}

Alertness is related to the LC-NE system (Posner \& Petersen, 1990). The LC is located in the brain stem and has numerous projections throughout the central nervous system, serving as the main NE production source. AstonJones and Cohen (2005) presented a theory that suggests two different modes of activity of the LC: tonic and phasic. In the tonic mode, LC neurons fire constantly, and this form of activity may be efficient during exploration for new rewards. The rationale is that when exploring for new rewards, one needs to be more sensitive to all surrounding stimuli, which is achieved by a constant release of NE. In contrast, the phasic firing mode elicits specific activation only for rewarding targets, but not for distractors. The shift between the two modes is thought to be modulated by the anterior cingulate and orbitofrontal cortices, both of which are considered to be involved in monitoring of task-related achievements provided by external feedback. Most studies examining the influence of alertness on cognitive abilities have used phasic manipulation of alertness, by introducing an alerting cue (Callejas, Lupiáñez, \& Tudela, 2004; Fan, McCandliss, Fossella, Flombaum, \& Posner, 2005; Fernandez-Duque \& Posner, 1997; Posner \& Bois, 1971). For instance, Fan, McCandliss, Sommer, Raz, and Posner (2002) devised the attentional network task (ANT), in which three attentional systems (alertness, orienting, and executive attention) can be measured using the same procedure. Fan et al. (2002) employed the ANT to study the three attentional systems and suggested that they are functionally independent (Fan et al., 2002) and involve separate neural structures (Fan et al., 2005). In contrast, Callejas et al. used a factorial design to explore the interactions between the attentional systems and suggested a functional interaction between them.

The theory put forward by Aston-Jones and Cohen (2005) may offer a more comprehensive involvement of the LC-NE system in cognitive effects. It is possible that different tasks activate different modes of LC-NE activity. Recently, several researchers suggested that the LC-NE system is involved in attentional effects that have traditionally been considered a product of attentional control. For instance, Nieuwenhuis, Gilzenrat, Holmes, and Cohen (2005) argued that the attentional blink (a temporary deficit in processing of a target stimulus following successful processing of a previous target) is a result of a refractorylike period caused by local NE release within the LC, in response to the first target. According to Verguts and Notebaert (2009), the Graton effect (smaller congruency effect following an incongruent trial), which is usually considered a result of attentional control, is actually a product of an interaction between binding processes and activation of the LC-NE system (see also Tzelgov \& Cohen Kadosh, 2009). According to this account, there is a phasic burst of NE during incongruent trials that increases Hebbian learning. Although these studies provide important theoretical arguments for the involvement of the LC-NE system in attentional control, they do not provide measures of the LC-NE activity during task performance and, thus, remain speculative.

\section{Involvement of the $\mathrm{LC}-\mathrm{NE}$ system in reflexive orienting of attention}

It has been demonstrated that simple discrimination tasks (e.g., discriminating between horizontal and vertical lines) produce phasic firing of the LC in nonhuman primates (Aston-Jones, Rajkowski, Kubiak, \& Alexinsky, 1994; Clayton, Rajkowski, Cohen, \& Aston-Jones, 2004). It has also been suggested that the phasic firing mode promotes focused selective attention and the tonic firing mode promotes scanning, labile attention (Aston-Jones, Rajkowski, \& Cohen, 2000). As was mentioned earlier, the onset of IOR appears later in discrimination tasks than in detection tasks. Gabay and Henik (2010) suggested that different modes of activity of the LC-NE system are employed during these different attentional tasks. Specifically, during a demanding discrimination task, the LCNE system functions in a phasic mode, since more 
resources are required in order to process the target and no resources are spared to process irrelevant information. In contrast, during an easier detection/localization task, the LC-NE system is activated in a tonic firing mode. According to Aston-Jones and Cohen (2005), the phasic mode also acts as a temporal filter for distractors; hence, in this mode, the LC-NE system releases lower levels of tonic NE during the presentation of distractors. In our opinion, the nonpredictive cue in an exogenous orienting task may be considered to be a distractor. If our hypothesis is correct, the reduced tonic firing of the LC at cue presentation can account for the delayed appearance of IOR in discrimination tasks. Such lower levels of NE during the presentation of the cue might slow down its processing and the attentional effects associated with its appearance (e.g., IOR). Aston-Jones and Cohen suggested using pupil size as an indicator for LC-NE activity, since pupil diameter correlates with LC tonic activity in monkeys and with behavioral performance.

\section{Pupil size measurement}

On top of its elementary role in monitoring light intensity levels, pupil size has primarily been considered as an indicator for mental effort (Kahneman \& Beatty, 1966; for a review, see Beatty, 1982). Pupil size is influenced by task difficulty in tasks involving language, reasoning, and perception. However, pupil size did not become a standard measure in psychological research, mainly due to the pupillary light reflex (greater intensity of light causes the pupil to contract). During visual experiments, the experimental conditions have often been confounded with the visual properties of the stimuli. For this reason, most studies involving pupil size measurements have been conducted using the auditory modality.

As was mentioned earlier, Aston-Jones and Cohen (2005) suggested that pupil size can be used as a measure of LC-NE activity. In this context, it is important to note that (1) LC-NE activation is more temporally related to the behavioral response than to the presentation of the target stimulus itself (Rajkowski, Majczynski, Clayton, \& AstonJones, 2004), and (2) it was demonstrated that changes in alertness are represented only by phasic changes in pupil size, and not by baseline pupil size level (Beatty, 1982). These findings suggest that a pupillary response related to LC-NE activity should be phasic and time-locked to the behavioral response.

The present work Our aim was to examine the involvement of alertness in orienting of attention. In particular, we explored the suggestion that in a more demanding task (discrimination), the LC-NE system is activated in a phasic mode and that, in an easy task (localization), it is activated in a tonic mode. We suggest that the difference in the mode of LC-NE activation can explain the differences in the time IOR appears between the two tasks. In the demanding task, the LC-NE system activates as a temporal filter and reduces NE levels during the appearance of the noninformative cue. Lower levels of NE during the time of cue appearance might delay cue processing and the attentional effects generated by it. As a result, IOR appears later in the more demanding task than in the less demanding task.

Here, we suggest a new approach for examining pupillary data, which overcomes the possible confounds elicited by the pupillary light reflex and other visual properties that may affect pupil measurement. Specifically, we suggest considering the use of pupillary data for comparison between two conditions: One condition should be used as a baseline (e.g., localization task), and the other as the test condition (e.g., discrimination task). It is important that the baseline and the test conditions be equated in terms of their visual properties and differ only in mental-processing requirements. By comparing the baseline score with the test score, we will get a pupillary response related only to the mental operations of interest. This is similar to Posner's (1978) suggestion for using RT to measure mental processes. In addition, since LC-NE activity is temporally related to the behavioral response, we employ a time-lock analysis of the data, in which we can compare the pupillary response time-locked to the cue, target, or response. As was suggested earlier, pupillary response as a result of $\mathrm{LC}-\mathrm{NE}$ activity should be presented time-locked to the response, but not to the cue or target appearance (Aston-Jones \& Cohen, 2005).

\section{Method}

Participants were randomly assigned to one of two groups. One group performed a localization task, and the other a discrimination task. The visual presentation of both tasks was identical; the only difference was the instructions participants received. In the localization group, participants were asked to respond according to the location of the target; in the discrimination task, participants responded to target identity.

\section{Participants}

Forty participants from Ben-Gurion University of the Negev participated in the experiment in exchange for course credit. All experimental procedures were approved by the ethics committee of the Psychology Department at Ben-Gurion University of the Negev. 
Apparatus and stimuli

The stimuli were white figures, on a black background, consisting of a fixation plus (which subtended a visual angle of $0.7^{\circ}$ ) at the center of the computer screen and three square boxes $\left(3^{\circ}\right.$ each side $)$ - one at the center of the screen, and the center of the other boxes $9.5^{\circ}$ to the left and right of the center of the screen. In each trial a target letter "O" or "Q" $\left(1^{\circ}\right)$ appeared in the center of one of the peripheral boxes. The target letter was preceded by a brightening of one of the two peripheral boxes, which was accomplished by widening the box's contour from $0.1^{\circ}$ to $0.5^{\circ}$. In the localization task, participants responded to the target by pressing one of two buttons on a keyboard according to the target location ( $\mathrm{Z}$ for targets appearing in the left box and $M$ for targets in the right box). In the discrimination task, participants responded by pressing the same buttons ( $\mathrm{Z}$ or $\mathrm{M})$ according to the target identity ( $\mathrm{O}$ or Q). Key assignment to target identity was counterbalanced between participants. A video-based desktop-mounted eyetracker (Eye Link1000, SR Research, Ontario, Canada) with a sampling rate of $250 \mathrm{~Hz}$ was used for recording eye movements and pupil dimensions. We used built-in programs provided with the eye tracker for calibration and validation purposes ( 9 points in a random sequence). All the data analyzed in the present article were obtained from recordings with an average Cartesian prediction error of less than $1^{\circ}$ during the validation procedures.

Procedure

Participants were tested in a dimly illuminated room. They were seated $57 \mathrm{~cm}$ from the computer monitor. Participants were instructed to maintain fixation on the fixation point throughout the experiment and to avoid blinking during the experimental trial (they were asked to blink between trials). If an eye movement or a blink were detected during the first $500 \mathrm{~ms}$ of the trial (in which a fixation plus was presented), an error sound was played, and the trial was restarted. If an eye movement or a blink were detected during the remainder of the trial, an error sound was played, and the trial was aborted. Participants were instructed to press one of two possible buttons ( $\mathrm{M}$ or $\mathrm{Z}$ buttons on a keyboard) as fast as possible when the target letter appeared, but to avoid false responses. Participants were also informed that the peripheral cue was not informative as to where the target would appear. Each trial began with the participant's pressing on the space bar, which initiated a drift correction and allowed time for blinks. This was followed by the appearance of a fixation plus, which was present throughout the experiment. One thousand five hundred $\mathrm{ms}$ after the beginning of a trial, one of two peripheral boxes brightened for $100 \mathrm{~ms}$ (the cue). One hundred ms, $400 \mathrm{~ms}, 700 \mathrm{~ms}$ or $1,000 \mathrm{~ms}$ after the onset of the cue (SOA), the target letter appeared in one of the peripheral boxes and remained in view until the participant responded, but not longer than 2,500 ms. After participants responded to the target, there was an intertrial interval of $1,000 \mathrm{~ms}$. No target appeared on 24 trials (i.e., catch trials), and participants were required not to respond. Each participant had 32 practice trials before the experiment began. The experiment contained 280 trials (divided into four blocks), of which 128 were valid and 128 invalid trials (32 trials for every validity and SOA condition).

\section{Results}

RT analyses

Trials on which the participants did not maintain fixation or blinked ( $9 \%$ of the trials for the localization task and $12 \%$ for the discrimination task), or responded incorrectly (less than $1 \%$ of the data for both tasks), too quickly ( $\leq 100 \mathrm{~ms}$ : no such responses were made), or too slowly ( $\geq 1,000 \mathrm{~ms}$; less than $1 \%$ for both tasks) were excluded from the analysis. During catch trials, subjects made fewer than $1 \%$ false responses for both tasks.

RT as a function of cue validity and SOA for both groups are presented in Fig. 1. As can be seen, the pattern of results fits the common finding that IOR appears earlier
Fig. 1 Response time (RT) as a function of task, stimulus onset asynchrony (SOA), and validity
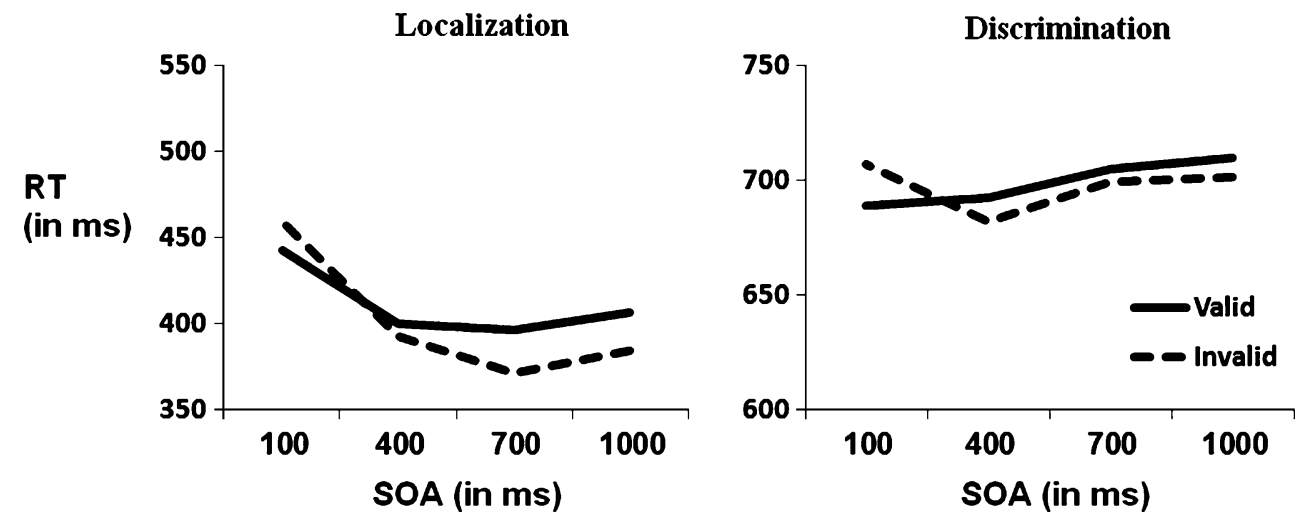
in detection than in discrimination tasks (Lupiáñez et al., 1997). We conducted two analyses of variance (ANOVAs) with $\operatorname{SOA}(100,400,700$, and 1,000 ms) and validity (valid, invalid) for every task. First, we will describe the ANOVA for the localization task and will continue by describing the ANOVA for the discrimination task. For the localization task, there was a significant main effect of SOA, $F(3,57)=$ $54.9, p<.001$, which indicated longer RT at the first SOA than at later SOAs. The interaction of SOA and validity was also significant, $F(3,57)=8.6, p<.001$. In order to study the time course of IOR, we examined the validity effect for every SOA, $F(1,19)=3.9, p=.06 ; F(1,19)=1.1$, n.s.; $F(1,19)=14.8, p<.01$; and $F(1,19)=6.6, p<.05$; for 100 -, $400-, 700-$, and 1,000-ms SOAs, respectively. A validity effect was marginally significant at the first SOA, and IOR was significant for the 700- and 1,000-ms SOAs. For the discrimination task, no main effects were significant. The interaction of SOA and validity was marginally significant, $F(3,57)=2.4, p=.07$. In order to examine this interaction, we studied the time course of IOR by examining the validity effect for every SOA, $F(1,19)=4.1, p=.05 ; F(1,19)=1.6$, n.s.; $F(1,19)<1$, n.s.; $F(1,19)<1$, n.s.; for $100-, 400-, 700-$, and 1,000-ms SOAs, respectively. There was a validity effect in the first SOA, but no IOR was apparent. A validity effect was presented at the shortest SOA, and IOR appeared at the third SOA in the localization task, but in contrast, in the discrimination task, the validity effect was present only in the first, shortest SOA and then was eliminated. Lupiáñez et al. (1997) demonstrated that IOR appears later in discrimination than in detection tasks. Although in Lupiáñez et al.'s work IOR was apparent at the 700-ms SOA in a discrimination task, our participants did not present a significant IOR even at
$1,000 \mathrm{~ms}$. A possible methodological difference between the tasks that might explain this difference is the experimental pace. In our experiment, every trial started after the participant pressed the space bar (which indicated that he or she was ready to begin the next trial). In Lupiáñez et al.'s experiment, a trial began immediately after the participants' keypress on the previous trial. Although IOR did not appear in the discrimination task, the disappearance of the validity effect at the second SOA might indicate the existence of an inhibitory influence (IOR).

\section{Pupillometry data}

We analyzed the pupil response time when locked to three events: cue, target, and manual response. Pupil size measurements were taken every $4 \mathrm{~ms}$, starting at $400 \mathrm{~ms}$ before the event and ending $400 \mathrm{~ms}$ after its termination. For each subject and event, we calculated mean pupil size (across trials; 200 segments per person). In order to explore the phasic changes in pupil size, we removed the slow linear changes in pupil size by calculating a regression line of pupil size on time segments for every subject and event and subtracting the actual pupil size from the predicted pupil size. Figure 2 presents the difference in pupil size between the two tasks (pupil size during discrimination minus pupil size during localization), time-locked to the three events within the trial (cue, target, and response) after the elimination of the linear trends. As can be seen, there is a phasic dilation of pupil size (peaking $200 \mathrm{~ms}$ after the event) only when the analysis is time-locked to the response. This effect corresponds to what would be expected for a phasic activation of the LC-NE system.
Fig. 2 Changes in pupil size time-locked to cue, target, or behavioral response.

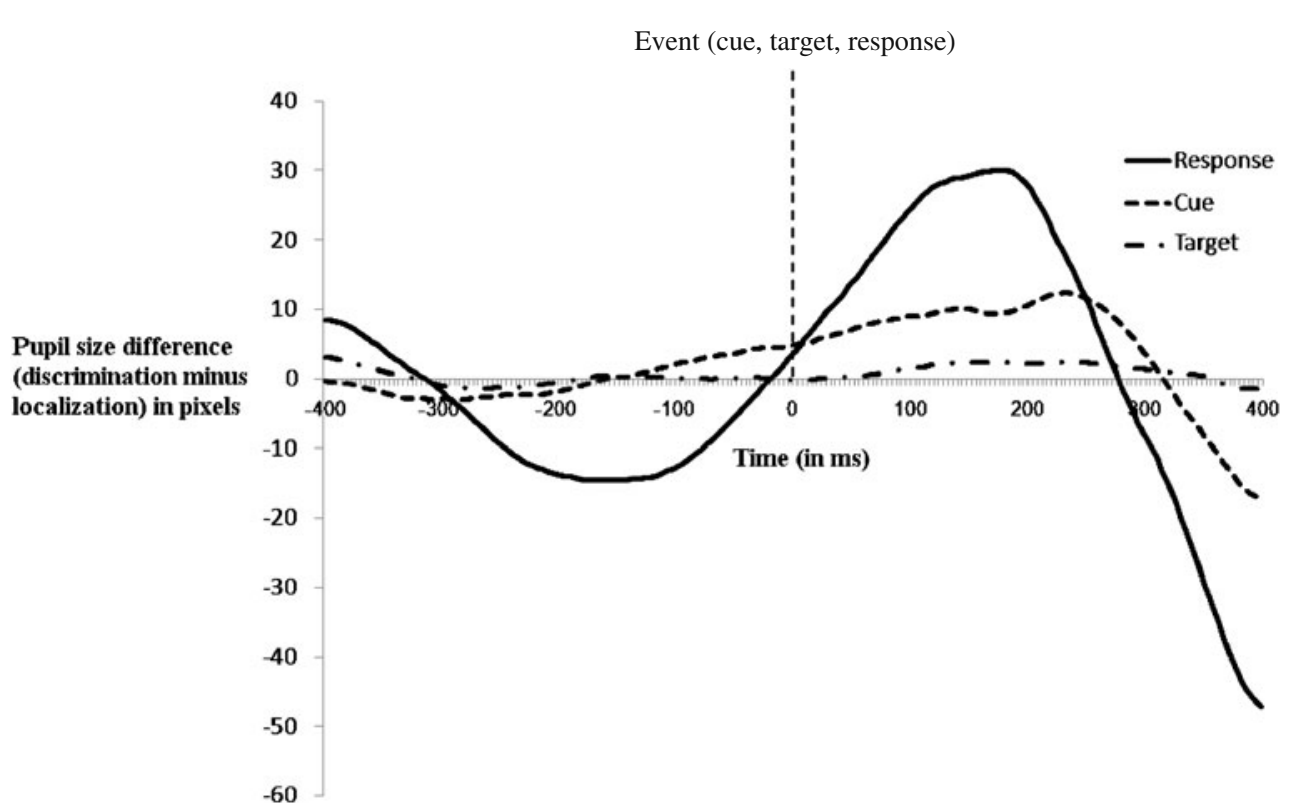


We examined the dynamics of the pupil size along the task. This was done separately for each of the three timelocked time courses. For each event (cue, target, and manual response) depicted in Fig. 2, we divided the time course into eight bins of $100 \mathrm{~ms}$ long: four bins preceding the onset of the event (1-4) and four following the event (5-8). For each subject, we calculated the mean pupil size (with the linear component partialled out) for each of the eight bins. These values were subjected to a two-way ANOVA (task $\times$ bin) for each of the events.

The ANOVA, time-locked to the response, revealed a significant main effect of bin, $F(7,266)=3.2, p<.01$, and a significant bin $\times$ task interaction, $F(7,266)=2.2, p<.05$. To examine this interaction, we compared the difference between tasks (discrimination and localization) for consecutive bins (a total of seven comparisons). These analyses indicated a significant change in pupil size when comparing bins 4 with 5 and 5 with $6, F(1,38)=14.2, p<.01$, and $F(1,38)=14.6, p<.01$, respectively. This indicates a change in pupil size between the two tasks that emerges just after a response was made.

An ANOVA of the time course locked to the target revealed only a main effect of bin, $F(7,266)=47.4, p<$ .01 ; the interaction was not significant, $F(7,266)<1$. Finally, similar to the target time-lock analysis, the ANOVA of the time course locked to the cue revealed only a main effect of bin, $F(7,266)=128.5, p<.01$, and a nonsignificant interaction, $F(7,266)<1$.

We found an interaction between bin and task for the response time-lock analysis. The two tasks differ in general RT:Localization is faster. In order to examine the influence of RT on the response time-lock analysis, we divided the participants in the two groups into four quartiles (slowest, slow, fast, and fastest participants) of response latency and added this (quartile) to the ANOVA. The interaction between quartile, task, and bin was not significant, $F<1$. This indicates that response latency did not influence our results.

Correlation between pupil size and IOR According to our hypothesis, the attentional effects produced by the nonpredictive cue are related to the amount of LC-NE activation during the presentation of the cue. We examined the correlations between the validity effect at every SOA and pupil size at the time of target appearance for the localization task, $r=.08, .36, .58$, and .38 for 100,400 , 700 , and $1,000 \mathrm{~ms}$, respectively, and for the discrimination task, $r=.02, .06,-.12$, and .32 , for $100,400,700$, and $1,000 \mathrm{~ms}$, respectively. We conducted a stepwise regression analysis with the validity effect for every SOA as the independent variable and pupil size as the dependent variable, for every task separately. For the localization task, the regression equation was significant $(R=.58$, adjusted .34). Only the third SOA validity was included in the model, $t=3, p<.0 ; \beta=.58$, (see Fig. 3). Since we wanted to examine the relation between pupil size and IOR, we divided the discrimination group into two groups of participants according to their pattern of results. Ten participants presented IOR at the third SOA, and $10 \mathrm{did}$ not. We created a dummy variable that distinguished between these two groups. We conducted a stepwise regression analysis with (1) validity effect (for the four SOAs), (2) IOR presence (IOR present or absent), and (3) interaction between the validity effects and the IOR presence (for the four SOAs). The regression equation was significant, $R=.44$, adjusted .2. Only the interaction between IOR presence and validity effect of the third SOA was included in the model, $t=2.1, p<.05$. The interaction was a product of a difference in the directionality of the correlation between pupil size and validity effect between participants that presented IOR (positive correlation; $r=$ .43 ) to those who did not (negative correlation; $r=.46$ ).

\section{Discussion}

In this experiment, we demonstrated that there is a phasic dilation of the pupil during discrimination tasks, as compared with localization tasks, time-locked to the behavioral response. We also found a correlation between pupil size and IOR. The present study is the first to measure LC-NE activity mode using pupillary response. Our specific design enables us to avoid the confound of the pupillary light reflex and other visual properties that might influence pupil measurement.

As was noted earlier, it was originally assumed that IOR does not appear in discrimination tasks (Terry et al., 1994), although a later work by Lupiáñez et al. (1997) showed that IOR appears later in discrimination than in detection tasks. Several theoretical suggestions were proposed in order to

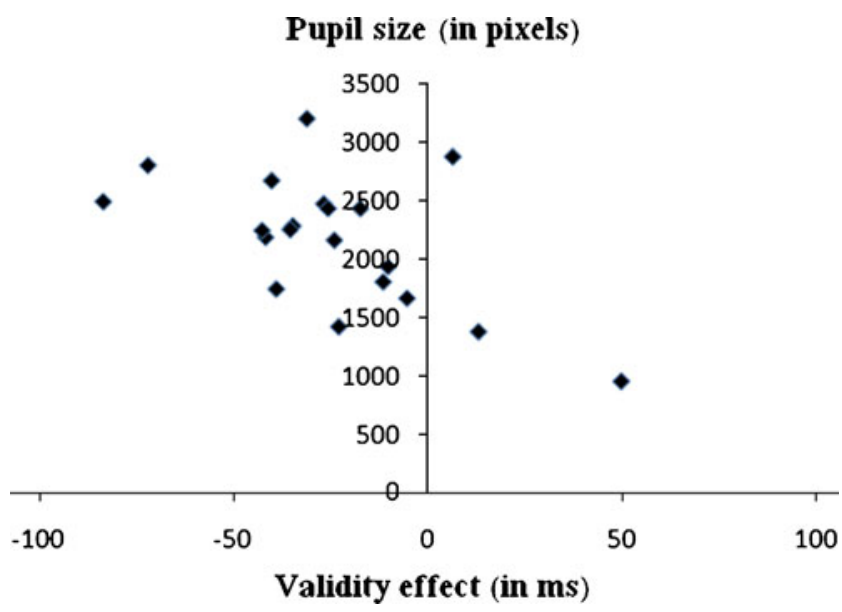

Fig. 3 Relation between pupil size and validity effect at the third stimulus onset asynchrony 
explain the difference of the time course between the two tasks. Egly and Homa (1991) suggested that in an easy detection task, focal attention is not involved, since it is not necessary in order to make an accurate decision. Klein (2000) suggested that in a more difficult task (discrimination), participants allocate more attention to all stimuli (target and cue), and the longer focus of attention at the cued location delays the appearance of IOR. Finally, Gabay and Henik (2010) suggested that the different activity modes of the LC-NE system are used for different attentional tasks. Accordingly, in this study, we demonstrated that in more demanding tasks (discrimination), the LC-NE system is activated in a phasic mode, and in easier tasks (localization/ detection), the LC-NE system is activated in a tonic mode. Due to the activation mode employed, lower NE is released during the appearance of the cue in the harder discrimination tasks. This, in turn, slows the processing of the cue and the attentional effects generated by it.

Aston-Jones and Cohen (2005) suggested that the phasic firing mode of the LC-NE system also acts as a temporal filter to distractors. In this case, in a more demanding task, the nonpredictive cue processing might be filtered by the reduced NE levels at the time of its appearance. In an easier task in which the LC-NE system is activated in a tonic mode, higher levels of NE are present at the time of cue presentation, and IOR appears earlier. This suggestion is strengthened by our finding that IOR magnitude was correlated to pupil size at the time of cue appearance. This finding also indicates that the processing of the cue, and not the processing of the target, influences IOR magnitude.

A direct measurement of LC-NE activity in humans requires invasive methods. Therefore, the link between $\mathrm{LC}-$ $\mathrm{NE}$ and pupil size is still speculative in humans; however, ample evidence from the monkey's brain does support this connection. Future research should further examine the relation between pupil size and LC-NE activity in humans.

Our work joins several recent studies that give the alerting system a central role in attentional effects that so far have been related to cognitive control (Nieuwenhuis et al., 2005; Verguts \& Notebaert, 2009).

Authors Note We would like to thank Dr. Galia Avidan for her helpful comments on early versions of the manuscript and also for providing technical support.

\section{References}

Aston-Jones, G., \& Cohen, J. D. (2005). An integrative theory of locus coeruleus-norepinephrine function: Adaptive gain and optimal performance. Annual Review of Neuroscience, 28, 403-450.

Aston-Jones, G., Rajkowski, J., \& Cohen, J. (2000). Locus coeruleus and regulation of behavioral flexibility and attention. Progress in Brain Research, 126, 165-182.
Aston-Jones, G., Rajkowski, J., Kubiak, P., \& Alexinsky, T. (1994). Locus coeruleus neurons in monkey are selectively activated by attended cues in a vigilance task. The Journal of Neuroscience, $14,4467-4480$.

Beatty, J. (1982). Task-evoked pupillary responses, processing load, and the structure of processing resources. Psychological Bulletin, 91, 276-292.

Berger, A., Henik, A., \& Rafal, R. (2005). Competition between endogenous and exogenous orienting of visual attention. Journal of Experimental Psychology: General, 134, 207-221.

Callejas, A., Lupiáñez, J., \& Tudela, P. (2004). The three attentional networks: On their independence and interactions. Brain and Cognition, 54, 225-227.

Clayton, E. C., Rajkowski, J., Cohen, J. D., \& Aston-Jones, G. (2004). Phasic activation of monkey locus ceruleus neurons by simple decisions in a forced-choice task. The Journal of Neuroscience, 24, 9914-9920.

Egly, R., \& Homa, D. (1991). Reallocation of visual attention. Journal of Experimental Psychology: Human Perception and Performance, 17, 142-159.

Fan, J., McCandliss, B. D., Fossella, J., Flombaum, J. I., \& Posner, M. I. (2005). The activation of attentional networks. Neuroimage, 26, 471-479.

Fan, J., McCandliss, B. D., Sommer, T., Raz, A., \& Posner, M. I. (2002). Testing the efficiency and independence of attentional networks. Journal of Cognitive Neuroscience, 14, 340-347.

Fernandez-Duque, D., \& Posner, M. I. (1997). Relating the mechanisms of orienting and alerting. Neuropsychologia, 35, $477-486$.

Gabay, S., \& Henik, A. (2010). Temporal expectancy modulates inhibition of return in a discrimination task. Psychonomic Bulletin \& Review, 17, 47-51.

Kahneman, D., \& Beatty, J. (1966). Pupil diameter and load on memory. Science, 154, 1583-1585.

Klein, R. (2000). Inhibition of return. Trends in Cognitive Sciences, 4, $138-146$

Lupiáñez, J., Milan, E. G., Tornay, F. J., Madrid, E., \& Tudela, P. (1997). Does IOR occur in discrimination tasks? Yes, it does, but later. Perception \& Psychophysics, 59, 1241-1254.

Nieuwenhuis, S., Gilzenrat, M. S., Holmes, B. D., \& Cohen, J. D. (2005). The role of the locus coeruleus in mediating the attentional blink: A neurocomputational theory. Journal of Experimental Psychology: General, 134, 291-307.

Posner, M. I. (1978). Chronometric explorations of mind. Hillsdale: Erlbaum

Posner, M. I., \& Bois, S. J. (1971). Components of attention. Psychological Review, 78, 391-408.

Posner, M. I., \& Cohen, Y. P. C. (1984). Components of visual orienting. In H. Bouma \& D. G. Bouwhuis (Eds.), Attention and performance X: Control of language processes (pp. 531-556). Hillsdale: Erlbaum.

Posner, M. I., \& Petersen, S. E. (1990). The attention system of the human brain. Annual Review of Neuroscience, 13, 25-42.

Rajkowski, J., Majczynski, H., Clayton, E., \& Aston-Jones, G. (2004). Activation of monkey locus coeruleus neurons varies with difficulty and behavioral performance in a target detection task. Journal of Neurophysiology, 92, 361-371.

Terry, K. M., Valdes, L. A., \& Neill, W. T. (1994). Does "inhibition of return" occur in discrimination tasks? Perception \& Psychophysics, 55, 279-286.

Tzelgov, J., \& Cohen Kadosh, R. (2009). From automaticity to control in bilinguals. Trends in Cognitive Sciences, 13, 455.

Verguts, T., \& Notebaert, W. (2009). Adaptation by binding: A learning account of cognitive control. Trends in Cognitive Sciences, 13, 252-257. 